\title{
Concurrent Potentiometric and Amperometric Sensing with Shared Reference Electrodes
}

\author{
Daryl Ma, Student Member, IEEE, Sara S. Ghoreishizadeh, Member, IEEE, \\ and Pantelis Georgiou, Senior Member, IEEE
}

\begin{abstract}
Potentiometry and amperometry are the two most common electrochemical sensing methods. They are conventionally performed at different times, although new applications are emerging that require their simultaneous usage in a single electrochemical cell. This paper investigates the feasibility and potential drawbacks of such a setup. We use a potentiometric and an amperometric sensor to compare their output signals when they are used individually, as well as when they are combined together with a shared reference electrode. Our results in particular show that potentiometric readings with a shared reference electrode show a high correlation of 0.9981 with conventional potentiometry. In the case of amperometric sensing, the cross correlation of the simultaneous versus individual measurement is 0.9959 . Furthermore, we also demonstrate concurrent measurement for potentiometry in the presence of cell current through the design of innovative test systems. This is done through measuring both varying $\mathrm{pH}$

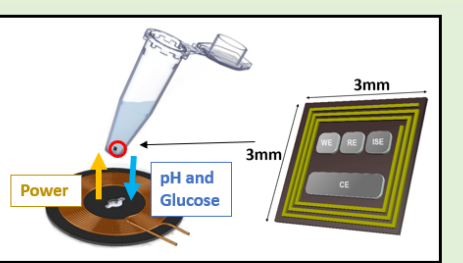

(A) Potential application of concurrent sensing of amperometry (Glucose) and potentiometry ( $\mathrm{pH})$
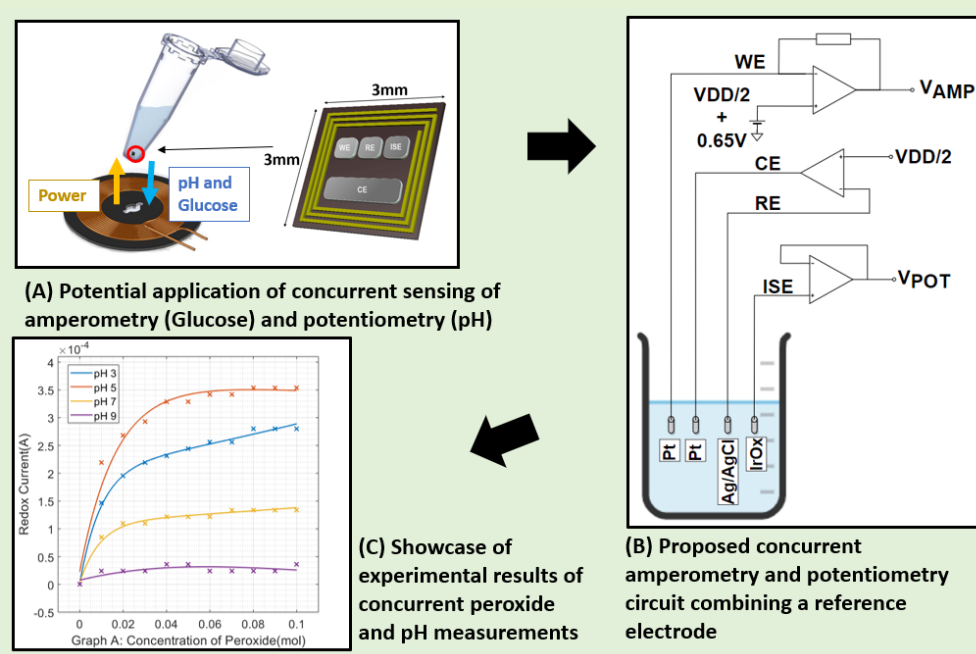

(B) Proposed concurrent amperometry and potentiometry circuit combining a reference electrode values and varying concentrations of $\mathrm{H}_{2} \mathrm{O}_{2}$ to showcase the operation of the circuit.

Index Terms-Electrochemical Sensing, Amperometry, Potentiometry, Concurrent Sensing, Iridium Oxide Electrodes, Experimental Design
\end{abstract}

\section{INTRODUCTION}

$\mathbf{E}$ LECTROCHEMICAL biosensors have made enormous progress in recent years due to their high sensitivity, specificity and their ability for rapid and inexpensive detection in small sample volumes [1]. Moreover, they lend themselves for integration into lab-on-chip platforms and allows for the monitoring of the concentration of target analytes in real time [2], qualities that are further highlighted in emerging health crises such as infectious diseases [3].

The emergence of ion-sensitive electrodes with low detection limits up to ultra trace levels have given rise to the importance of potentiometric sensing [4]. With ongoing research on miniaturising these electrodes further, potentiometry allows for the possibility of low ion measurement values in tiny sample sizes for ions such as potassium, sodium, $\mathrm{pH}$ values and even

This manuscript was submitted on the 20th of August 2020.

Daryl Ma and Pantelis Georgiou are with the Centre for Bio-Inspired Technology, Department of Electrical \& Electronic Engineering, Imperial College London, UK (Email: \{darylma, pantelis\}@imperial.ac.uk)

Sara Ghoreishizadeh is with the Aspire Centre for Rehabilitation and Assistive Technology, University College London, UK (Email: s.ghoreishizadeh@ucl.ac.uk)

* Sara Ghoreishizadeh and Pantelis Georgiou are co-senior authors of this work. bacteria or toxicities [5] [6].

On the other hand, amperometry is a measurement technique usually applied for the detection of biomolecules such as lactate, ATP, glucose and cholesterol [7]. In addition to this, it has also been extensively applied for gas sensors, most recently in the field of low-cost air quality control [8]. Amperometry is also particularly relevant in the field of glucose sensing, where numerous amperometric sensors are utilised [9].

The recent advent of electrochemical sensors integrated with complementary metal-oxide semiconductor (CMOS) technology allows for low-cost, portability and scalability at low noise and power levels. [10]. However, these integrated CMOS sensors comes at a cost of being sensitive to temperature, as well as having limited lifespans when used in the field due to material degradation [11]. Biofludic measurement is helpful for real-time detection of diseases [12]. In the case of infectious diseases, having the ability to self monitor diagnostics at home is especially useful [13]. One such biofluid that is inexpensive and non-invasive is saliva, which is emerging as an alternative to blood for state of health monitoring [14]. Several salivary biomolecules correlate with serum [15] - which makes it helpful for potential research fields in non-invasive health monitoring [16]. 
A key challenge in the measurement of biomolecules in saliva is the presence of interfering molecules. Additionally, changes in environmental parameters such as temperature and $\mathrm{pH}$ of saliva affects the electrochemical sensors [17]. Salivary $\mathrm{pH}$ follows a circadian rhythm that changes dependent on the body's dietary conditions, gastric acid reflux, diabetes or oral health situation [18]. Measuring electrochemical biomedical readings in tandem with $\mathrm{pH}$ measurements provides additional metrics helpful for improving the accuracy of the readings. Concurrent measurements of potentiometric and amperometric monitoring of various analytes for co-analysis allows for sensor calibration at real-time [19].

Moreover, the inherent differences of potentiometry and amperometry result in advantages and disadvantages for either technique [20]. In particular, Brunt et al. states that amperometry allows a faster measurement of glucose in flow cells, while the potentiometric technique provides a lower limit of detection. The input-output characteristics of the potentiometric and amperometric sensors are also different, with potentiometry having a logarithmic response while amperometry is linear. Having the possibility of utilising both techniques allows the advantages of either technique, and provide for a more versatile analysis.

Examples of systems capable of both amperometry and potentiometry include Sun et al. [21] and Jiang et al. [22], who describe reconfigurable circuits capable of operating in different modes for amperometry and potentiometry, however these are performed at different time periods.

This work investigates concurrent potentiometry and amperometry through the means of a shared reference electrode, and demonstrates the accuracy of potentiometry in the presence of a cell current based on tests designed for this purpose. This new configuration is henceforth referred to as the combined configuration. This configuration allows for the capture of instantaneous $\mathrm{pH}$ changes for accurate calibration and reliable analysis [23]. An empirical approach as to how concurrent sensing affects potentiometry is done through a comparison of a standard electrode configuration with the combined electrode configuration.

We will analyse how they affect the accuracy of the measurements. Their sensitivities are compared, and a correlation between the separate and combined configurations is obtained. $\mathrm{pH}$ and hydrogen peroxide $\left(\mathrm{H}_{2} \mathrm{O}_{2}\right)$ are selected as target analytes for potentiometric and amperometric measurements respectively, due to their importance in electrochemistry and the simplicity of the fabrication of the sensors.

A brief background into the theory behind electrode sensing for potentiometry and amperometry is provided in the next section. Section III presents a detailed explanation of the reference electrode operation as this is the key difference in this experiment. The materials and methods along with the experimental setup is presented in Section IV, while Section V covers the obtained results, followed by a detailed analysis in Section VI.Lastly, a final conclusion is given as well as future research directions.

\section{THEORETICAL BACKGROUND}

To describe the operation of potentiometry and amperometry, a background into the operation of electrodes and their equivalent circuits is provided. The sensing operation at the electrode as well as circuit level is then covered for both potentiometry and amperometry.

\section{A. Electrodes}

Two electrodes are employed in potentiometry, consisting of a sensing and reference electrode. The sensing electrode in this work is an ion-sensitive electrode (ISE), and serves to measure the ion concentration relative to a stable reference. The amperometric measurement typically includes three electrodes: Working (WE), Counter (CE) and a Reference electrode (RE). A potential is induced across the WE and RE, while the CE provides a steady current supply. Randles cell [24] is an equivalent circuit model for an electrode in an electrolyte and is illustrated in Fig. 1.

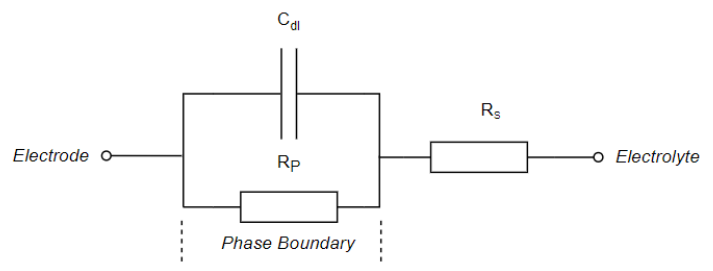

Fig. 1. Simplified Randles Half-Cell Model that describes the electrodeelectrolyte interface of a single electrode

$C_{d l}$ describes the double layer capacitance from the electrical alignment of water molecules near the electrode surface to the electrolyte. $R_{s}$ is the electrolyte resistance, which is affected by electrode material, electrode construction and tissue impedance. $R_{P}$ represents the charge transfer resistance at the phase boundary, which involves the electrode oxidation and reduction reactions [25].

It is not possible to measure the potential across the electrolyte and electrode, as there is no physical connection point at the electrolyte. Instead, this model describes the theoretical reactions across the electrolyte and phase boundary. In order to determine the potential across a half-cell, another electrode is required as a reference with a known potential to compare any measured potential to.

\section{B. Potentiometry}

As can be seen from Fig. 2, a junction potential arises due to the charges at the interface between an electrode and an electrolyte. Potentiometry is the measurement of this charge through the use of a high-impedance voltmeter between the sensing electrode and a RE, ensuring that no current passes through - leaving the circuit in equilibrium.

ISEs work specifically by allowing only the desired ions to be adsorbed upon the electrode surface. In particular, for metal oxide/metal oxide $\mathrm{pH}$ sensors such as the iridium oxide (IrOx) electrodes described in this work [26], this is dependent on the equilibrium between the oxide layers. This potential at the sensing electrode is related to the Nernst Equation for ISEs: 


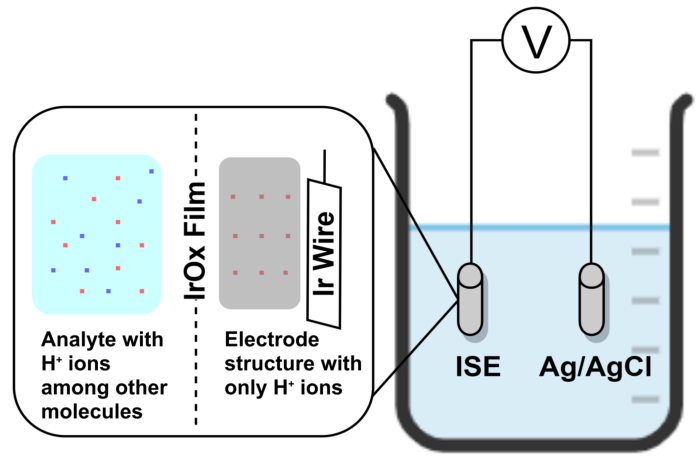

Fig. 2. Potentiometry set-up with a graphical description of the junction potential of an iridium oxide $\mathrm{pH}$ electrode

$$
E=E^{0}+\frac{2.303 R T}{n F} \log (A)
$$

where $\mathrm{E}$ is the total potential in $\mathrm{mV}$ between the sensing and reference electrodes, $E^{0}$ the constant based on the ISE/reference pair, $\mathrm{R}$ is the gas constant (8.314 Joule/degree/mol), T is the absolute temperature (298.15K @ $\left.25^{\circ} \mathrm{C}\right), \mathrm{n}$ the ion charge sign, $\mathrm{F}$ the Faraday Constant $(96.5 \mathrm{~K}$ Coulombs) and A refers to the reaction quotient, which in this case would be the concentration of ions in the solution [27].

In potentiometry, the circuit is kept at equilibrium and no current is flowing through the electrodes. When this equilibrium is disturbed through a variation in the potential, a current is produced based on Faraday's Law [28].

\section{Amperometry}

Amperometry involves the technique of applying the potentiostat to measure the concentration of a certain ion in a solution. Once a potential is induced across the RE and the WE, the detected ions diffuses towards the WE. These ions are then adsorbed onto the WE, where a redox reaction occurs. The products of the reaction then desorb away from the WE. To achieve ion-selectivity, a diffusion membrane can be introduced to limit the reaction for the desired ions [29]. The CE is utilised in this case to provide a steady current supply without affecting the potential difference - hence the term potentiostatic.

The analytical response of the sensor is the magnitude of the current between the WE and the CE. The potential of the WE is chosen such that the faradaic current is determined by diffusion instead of the kinetics of the redox reaction, which allows for a linear relation between the current and the concentration of the detection ion.

\section{Reference Electrode}

The reference electrode is an electrode that has a stable and well defined potential, where all other potentials in the circuit are referenced to. Standard Hydrogen Electrode (SHE), Saturated Calomel Electrode (SCE) or the Silver/Silver Chloride Electrode $(\mathrm{Ag} / \mathrm{AgCl})$ are the most commonly used reference electrodes.

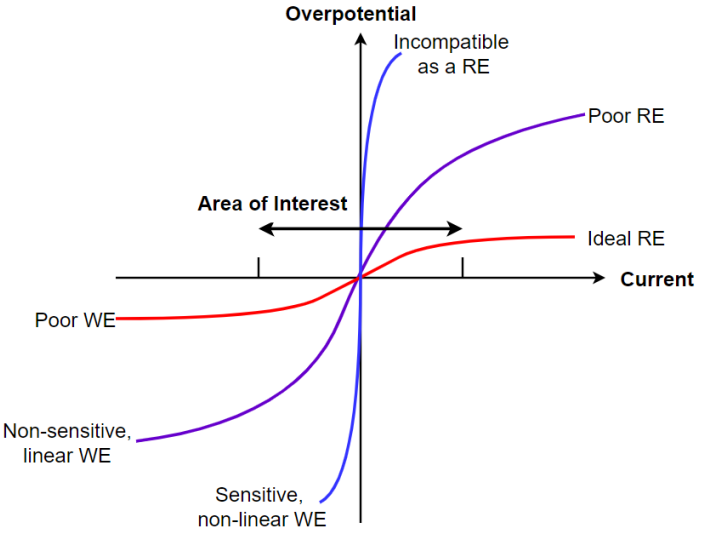

Fig. 3. I-V curves for various materials - Non-polarisable representing a good RE, with minimal potential change across a large range of current, as well as linear (Polarisable) or sensitive (Highly Polarisable) WEs. [30]

As mentioned, a current is produced when there is a variation in the potential. This current and voltage relationship causes electrode polarisation, which is the phenomena whereby the potential across the electrode-electrolyte interface varies with the current flow. Polarisation differs for various materials as shown in Fig. 3 - with the degree of polarisation being key to the type of electrode the material will be suitable for. Polarisation is affected by 3 factors: (1) Activation, whereby the potential difference depends upon the activation energy of the reaction; (2) Concentration, whereby the change in ion concentration varies the equilibrium potential of the reaction; (3) Ohmic, where a resistive drop across the electrolyte occurs due to the volume resistance [31]. Fig. 3 also displays the I-V curves for ideal REs or WEs. To measure the degree of polarisation, a potentiostat is utilised to maintain a fixed potential while measuring the current induced.

The requirements of a reference electrode are as follows [30]: (1) It must be non-polarisable and reversible with a high exchange current density. These allow the potential to remain constant for a large current range. (2) The reaction area should remain constant throughout the reaction, with the reaction not affecting the electrode area. (3) Lastly, the inner filling solution of the electrode should be saturated. This ensures that the concentration of the inner filling solution remains relatively stable even when the solvent evaporates - and leaves the potential constant. It also follows that a high saturation allows for a higher and stable exchange current density as shown in Fig. 3. This also allows the test solution to be separate from the inner filling solution, and hence the potential is as constant as possible.

Most of the characteristics of the RE such as being nonpolarisable and having a constant potential is shared across the potentiometric and amperometric techniques. However, a key difference lies in their electrical control requirements (i.e. associated circuit topologies). In potentiometry (see Fig. 4B), the RE is connected to a low-impedance output node of an op-amp. This is key for the RE to be driven by the op-amp to a desired reference voltage. In contrast, for amperometry (see Fig. 4A) the RE is connected to a high-impedance input node in a positive feedback configuration. This ensures zero-current 
flows into the RE. As current flows from the CE, this topology ensures that the current goes directly to the WE.

For the combined configuration, the RE is connected similarly to the amperometric RE - a high-impedance connection. Although the change of RE configuration does not affect the ISE enlivenment (because RE simply provides a reference point for the measurements), the voltage between the electrode and the electrolyte at the ISE may become affected due to the current flow induced by the amperometry. Ion flow across the solution might affect the concentration across the junction potential - leading to erratic readings. We expect this to be negligible for steady state measurements. We will explore the scale of this effect in this work through carefully designed experiments. Another potential issue is the possibility of the reference electrode voltage drifting due to the open-loop topology employed.

The effects of a controlled external current applied to potentiometric readings has been explored in [32] [33] [34]. The utilisation of external current has led to an improvement in the lower limit of detection for potentiometry [32] and [33]. A theoretical model described in [34] further expands upon the potential response of the ISE demonstrating that a controlledcurrent allowed for improved lower limits of detections for ISE.

The amperometric measurements should be similar across both the combined and separated topologies because the addition of a passive ISE will not impact the amperometric cell.

\section{ExPERIMENTAL DEsign AND Materials}

To compare the efficacy of using a shared reference electrode, the results for the classical measurement techniques will be compared to the results obtained for the combined reference electrode configuration.

The combined circuit consists of the amperometric circuit along with the ISE sensing op-amp of the potentiometric circuit. This combined configuration contains the ISE utilising the reference of the open loop circuit.

\section{A. Materials}

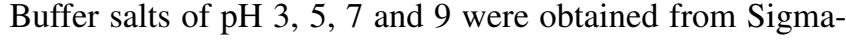
Aldrich to provide the calibration and tests for potentiometric measurements. $30 \% \mathrm{H}_{2} \mathrm{O}_{2}(1.11 \mathrm{~g} / \mathrm{cm} 3)$ from Fisher Bioreagents is used for amperometry.

$\mathrm{An} \mathrm{Ag} / \mathrm{AgCl}$ glass reference electrode with a porous teflon tip purchased from $\mathrm{CH}$ Instruments (CHI111) [35] is used as the reference electrode for the separate and the combined topologies. The 550 from Dropsens with a platinum CE and WE were used for amperometry [36].

Iridium Oxide electrodes have been chosen as the potentiometric sensing electrode for $\mathrm{pH}$. It has been shown to have a life span of up to 2.5 years [37] - which makes it particularly useful for ensuring sensitivity across the span of the experiment. These electrodes are fabricated for the experiments. Iridium wire (178 $\mu \mathrm{m}$ diameter, $99.9 \%$ purity) is obtained from ADVENT RM, along with sulphuric acid $(5 \% \mathrm{~V} / \mathrm{V})$ from Sigma Aldrich.

\section{B. Equipment}

A Sentron SI400 pH meter [38] with an accuracy of $0.01 \mathrm{pH}$ was used as a gold standard for $\mathrm{pH}$ measurement. A CH700E Potentistat from CH Instruments [39] is used for the fabrication of the $\mathrm{IrOx} \mathrm{pH}$ sensor. A Saleae logic analyzer with 8 inputs is used in tandem with multimeters to monitor the voltages from the various outputs of the circuit.

\section{IrOx Electrode Fabrication}

The IrOx based $\mathrm{pH}$ sensor is used due to their high sensitivity, stability and life time. We fabricated the IrOx electrode in house with the following recipe: Iridium wire (178 $\mu \mathrm{m}$ diameter, $99.9 \%$ purity) is oxidised through immersion in a sulfuric acid solution $(5 \% \mathrm{~V} / \mathrm{V})$. During the oxidation phase, the $\mathrm{Ag} / \mathrm{AgCl}$ glass reference electrode along with a platinum counter electrode are immersed inside the sulfuric acid solution. Repetitive cyclic voltammetries between $-0.2 \mathrm{~V}$ and $1.2 \mathrm{~V}$ at a $1.4 \mathrm{~V} / \mathrm{s}$ scan rate is performed on the three electrodes for 3 hours, with the iridium wire serving as the WE.

The oxidised wire is next calibrated in order to obtain the $\mathrm{pH}$ sensitivity. This is done through the use of $\mathrm{pH}$ buffers. The final fabricated wire is then soldered to a 28 AWG wire as a connection to the sensing circuitry. This connection is covered in Araldite epoxy and the entire surface sealed with a heat shrink to reduce the effect of any contamination of the electrode surface.

\section{Sensing Circuitry}

The configuration of electrodes and connection with the readout electronics for the separate and combined experiments is shown in Fig. 4. This is the overall experimental setup.

A custom-designed external circuit has been used in this work because the electronic instrumentation that is required to drive an electrochemical cell for concurrent potentiometric and amperometric measurements (including a shared reference electrode) is not available in current commercial potentiostats, and the reference electrode is unique. Moreover, the commercial electrochemical sensing equipment such as the $\mathrm{CHI} 700 \mathrm{E}$ provides access to the output nodes, but unfortunately does not provide access to node $\mathrm{V}_{a m p}$ in the potentiostat. This made it less convenient to test the unique sensing topologies.

The op-amp utilised for all circuits is the LMC6042 [40] from Texas Instruments. This op-amp is chosen for its ultralow input leakage current, which helps prevent any possible drift from the reference electrodes.

For the potentiometric circuit, a $\frac{V D D}{2}$ voltage is applied to the reference electrode to allow for a maximum input swing for the ISE detection range.

In the separate amperometric circuit (see Fig. 4A), a transimpedance amplifier is used to detect the current flowing into the WE. The $\mathrm{WE}$ is biased at $0.65 \mathrm{~V}$ higher than $\frac{V D D}{2}$ to activate the redox reaction of $\mathrm{H}_{2} \mathrm{O}_{2}$ [41], with the $\mathrm{CE}$ connected to the output of the op-amp to provide the current necessary for the RE to be held at the $\frac{V D D}{2}$ value. 


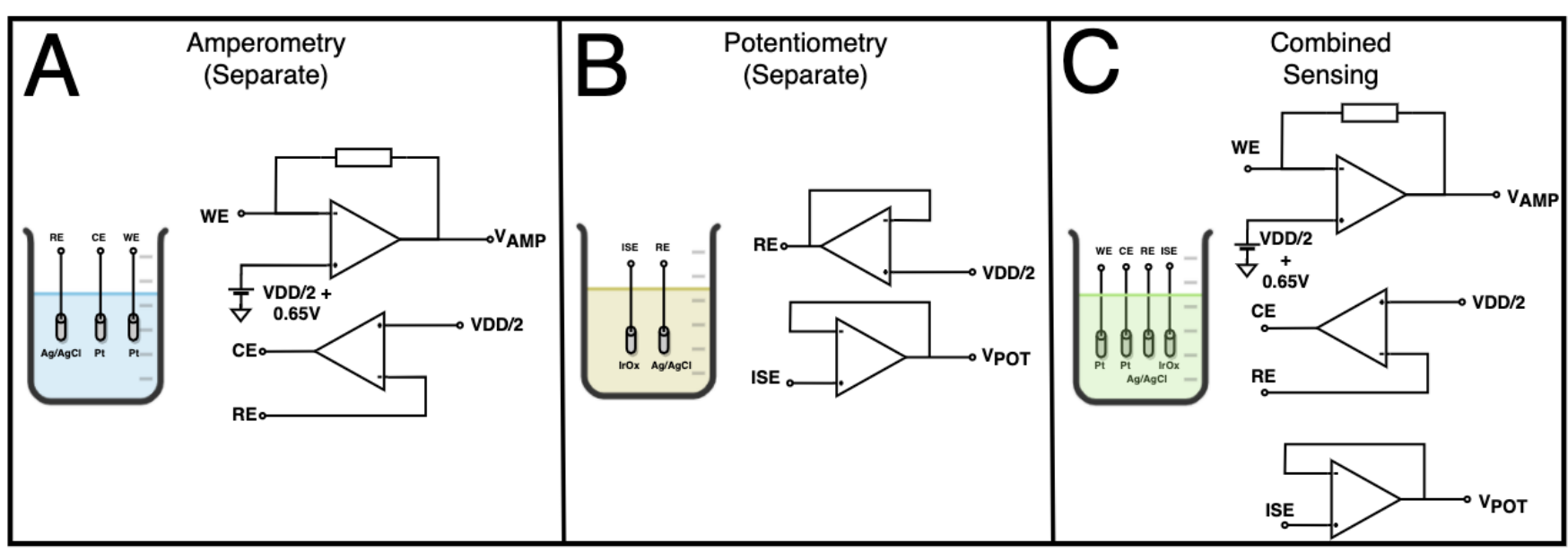

Fig. 4. Experiment Circuitry employed for this experiment with the separate potentiometric and amperometric modes along with the combined setup. The chosen electrodes along with their materials are also displayed. This is the overall experimental setup applied in this work, with the details on the chemical aspect of the experiments explained in Section IV.

\section{EXPERIMENTAL RESULTS}

\section{A. Combined vs separate measurement: sensor sensitivity and correlation}

1) Potentiometric: The aim of this experiment is to determine if there is any difference in the sensitivity of the $\mathrm{pH}$ sensor between zero and non-zero cell current scenarios. The protocol is as follows:

1) $20 \mathrm{ml}$ of $\mathrm{pH}$ buffer solutions prepared for $\mathrm{pH} 3,5,7$ and 9. All solutions are $0 \mathrm{mM}$ concentration of $\mathrm{H}_{2} \mathrm{O}_{2}$.

2) $\mathrm{V}_{\text {pot }}$ and $\mathrm{RE}$ were measured at all four $\mathrm{pH}$ buffer solutions with the setup of Fig. 4B.

3) The voltages at $\mathrm{V}_{\text {pot }}$ and RE were measured again this time using the setup shown in Fig. 4C.

4) Experiment repeated thrice to determine error ratio.

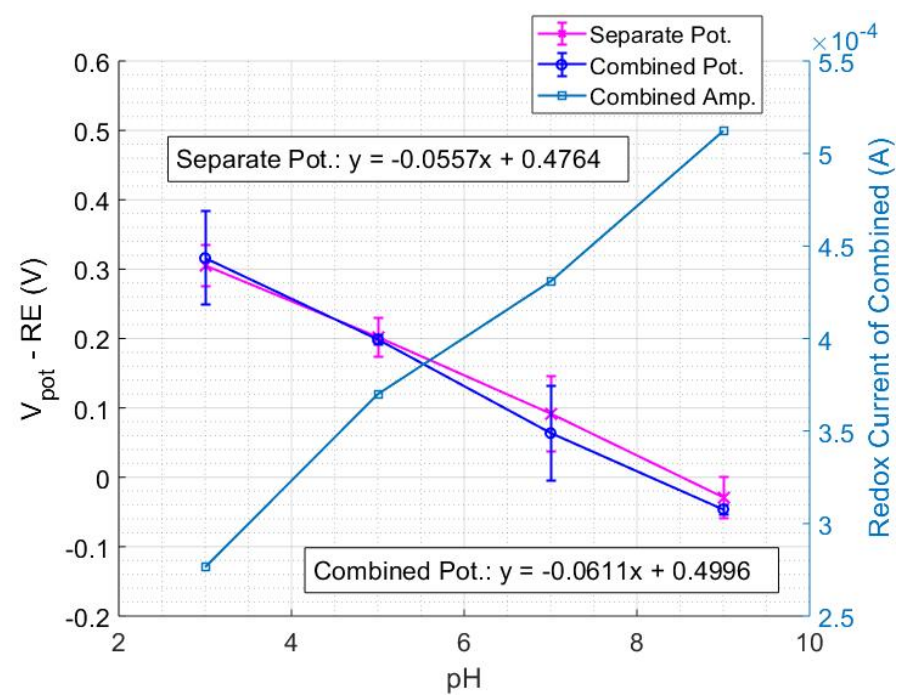

Fig. 5. Separate vs Combined potentiometric readings (error bars represent max and min values from three trials). Combined amperometric reading is shown on the plot, with the axis on the right. Cross correlation of 0.9981 observed. The entirety of this experiment is with no added $\mathrm{H}_{2} \mathrm{O}_{2}$, which means the cell current is entirely due to background current.
The measured potentiometric voltages together with the measured amperometric current in the combined setup are presented in Fig. 5. The error bars represent the max and min of the measured data points. The results for the combined potentiometry display a larger error ratio. A cross correlation of 0.9981 between both sets of results indicate a clear similarity between the separate and combined configurations. The amperometric current generated is purely from the $\mathrm{pH}$ buffer (i.e not due to $\mathrm{H}_{2} \mathrm{O}_{2}$ ), and shows an increment for higher $\mathrm{pH}$ buffers.

2) Amperometric: The purpose of this set of experiments is to determine the sensitivity of the amperometric sensor, as well as to determine the correlation between the separate and combined settings of the amperometric outputs. The protocols are:

1) $20 \mathrm{ml}$ of $\mathrm{pH} 3$ solution prepared on a magnetic stirrer (rpm 200)

2) Increasing concentrations of $0.01 \mathrm{M}$ of $\mathrm{H}_{2} \mathrm{O}_{2}$ is added to the solution.

3) Measurements of the cell current is taken for the separate configuration as shown in Fig. 4A for $\mathrm{V}_{a m p}, \mathrm{CE}$ and RE up to the full range of concentration of $0.1 \mathrm{M}$.

4) Measurements of the cell current is taken for the combined configuration (Fig. 4C) for $\mathrm{V}_{a m p}, \mathrm{CE}$ and RE up to $0.1 \mathrm{M}$.

5) Experiment repeated thrice to determine error ratio.

The amperometric measurements for both the separate and combined are presented in Fig. 6. A logarithmic fit is applied, with the max and min of the three experiments shown. The results show a high correlation of 0.9959 , which demonstrate that the amperometric measurement is unaffected by the addition of the ISE.

\section{B. Combined Configuration: Concurrent measurements for varying $\mathrm{pH}$ and $\mathrm{H}_{2} \mathrm{O}_{2}$ concentrations}

To determine whether potentiometric measurement shows a difference in the presence of a variable cell current, we 


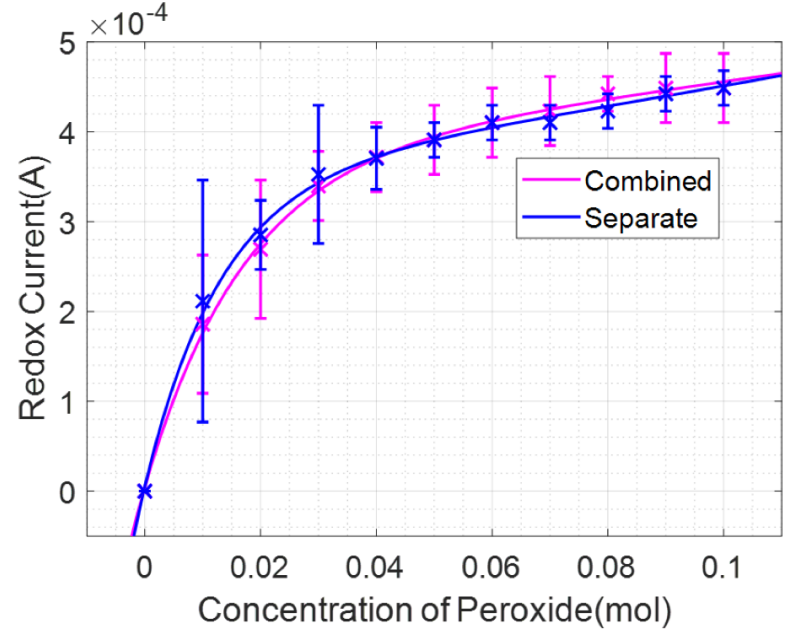

Fig. 6. Separate vs Combined amperometric readings averaged across three trials with a cross correlation of 0.9959 observed and background current removed. The maximum and minimum of the three measured samples are shown in the plot with a logarithmic fit. The tests are performed at a fixed $\mathrm{pH} 3$ buffer.

configure the analyte in the setup shown in Fig. 4C such that the amperometric current varies (by changing $\mathrm{H}_{2} \mathrm{O}_{2}$ concentration) for fixed $\mathrm{pH}$ buffers. To this aim we designed and conducted two experiments described in this section.

1) Amperometric Measurements: This experiment serves to showcase the varying cell current for varying $\mathrm{H}_{2} \mathrm{O}_{2}$ concentrations in different $\mathrm{pH}$ buffers. The protocol is as follows:

1) $20 \mathrm{ml}$ of $\mathrm{pH} \mathrm{3,5,7}$ and 9 solution prepared on a magnetic stirrer (rpm 200)

2) Increasing concentrations of $0.01 \mathrm{M}$ of $\mathrm{H}_{2} \mathrm{O}_{2}$ is added to the solution.

3) Sentron $\mathrm{pH}$ meter is used as the gold standard $\mathrm{pH}$ meter.

4) Measurements of amperometry taken for the combined configuration (see Fig. 4C) for $\mathrm{V}_{a m p}, \mathrm{CE}$ and $\mathrm{RE}$ for the full range of concentration as shown in Fig. 7A.

The measured results for amperometry are shown in Fig. 7A. All measurements were performed on the same day. The results show that the cell current variation differs for different $\mathrm{pH}$ buffers. This affirms that the cell current varies for increasing peroxide levels, although for lower $\mathrm{pH}$ levels $(3,5)$ the variation in the cell current is much higher as compared to the high $\mathrm{pH}$ levels $(7,9)$. With it being clear that there is a cell current generated from increasing peroxide levels, the next experiment would aim to determine if there is a variation in the potentiometric results after this cell current is added.

2) Potentiometric Measurements: This experiment seeks to observe the difference of the potentiometric readings in the event the the cell current is varied. This is done by varying the concentration of $\mathrm{H}_{2} \mathrm{O}_{2}$ in various $\mathrm{pH}$ buffers, and comparing them with $\mathrm{pH}$ measurements utilising a gold standard. The protocol is as follows:

1) $20 \mathrm{ml}$ of $\mathrm{pH} \mathrm{3,5,7}$ and 9 solution prepared on a magnetic stirrer (rpm 200)

2) Increasing concentrations of $0.01 \mathrm{M}$ of $\mathrm{H}_{2} \mathrm{O}_{2}$ is added to the solution.

3) Sentron $\mathrm{pH}$ meter is used to serve as the gold standard
$\mathrm{pH}$ value with readings taken for each concentration value.

4) Measurements of potentiometry taken for the combined configuration (Fig. 4C) for $\mathrm{V}_{\text {pot }}$ and RE for the full range of concentration as shown in Fig. 7B.

The results are shown in Fig. 7B. The measurements with the gold standard shows that the $\mathrm{pH}$ remains constant, and only changes slightly (up to 10\%) for higher concentrations of $\mathrm{H}_{2} \mathrm{O}_{2}$.

The difference between the gold standard and the IrOx electrode observed is shown in Table I and can be characterised as varying between a negative value for both $\mathrm{pH} 5$ and 7 and a positive value for $\mathrm{pH} 3$ and 9. This value for $\mathrm{pH} \mathrm{5,7}$ and 9 remain relative constant with variation around $\pm 0.4 \mathrm{pH}$.

TABLE I

THE MEAN AND STANDARD DEVIATION OF THE PH DIFFERENCE BETWEEN MEASURED ISE RESULTS AND CONTROL FOR VARIOUS PH VALUES (ISE-CONTROL)

\begin{tabular}{|c|c|c|}
\hline $\mathbf{p H}$ & $\begin{array}{c}\text { Mean of } \\
\text { pH difference }\end{array}$ & Standard Dev. \\
\hline 3 & 0.099 & 0.103 \\
\hline 5 & -0.129 & 0.0587 \\
\hline 7 & -0.175 & 0.0134 \\
\hline 9 & 0.398 & 0.0724 \\
\hline
\end{tabular}

\section{ANALYSIS AND DISCUSSION}

\section{A. Comparison of Separate vs Combined}

The sensitivity of the separate and combined potentiometric readings are $-0.0557 \mathrm{~V} / \mathrm{pH}$ and $-0.0661 \mathrm{~V} / \mathrm{pH}$ respectively as seen in Fig. 5. This indicates that the ISE sensor behaves similarly, and also demonstrates the feasibility of the use of potentiometric readings with a shared reference electrode. More importantly, the varying cell current (as measured by the amperometric sensor) showcases that even for increasing cell current for higher $\mathrm{pH}$ values, the combined potentiometric sensor is still capable of behaving ideally, albeit with an error ratio up to $26 \%$ higher. This can be seen at the $\mathrm{pH} 7$ readings, with the combined configuration having a slightly higher error ratio compared to the separate configuration. A cross correlation of 0.9981 particularly indicates the high statistical similarity in their trends.

As the separate and combined amperometric readings differ only through the addition of the ISE electrode, the results in Fig. 6 are as expected with both configurations following a similar trend with a slight offset in the current readings. The high cross correlation of 0.9959 between both the combined and separate amperometric readings leads to the conclusion that the amperometric sensors in both configurations have very similar trends.

\section{B. Potentiometry with Cell Current}

A particular challenge faced is to demonstrate the accuracy of the potentiometric readings in the presence of various levels of cell current. This is highly unconventional, and prior work in [32] and [33] has shown the possible effects of this cell current on enhancing potentiometry. In comparison, we are 

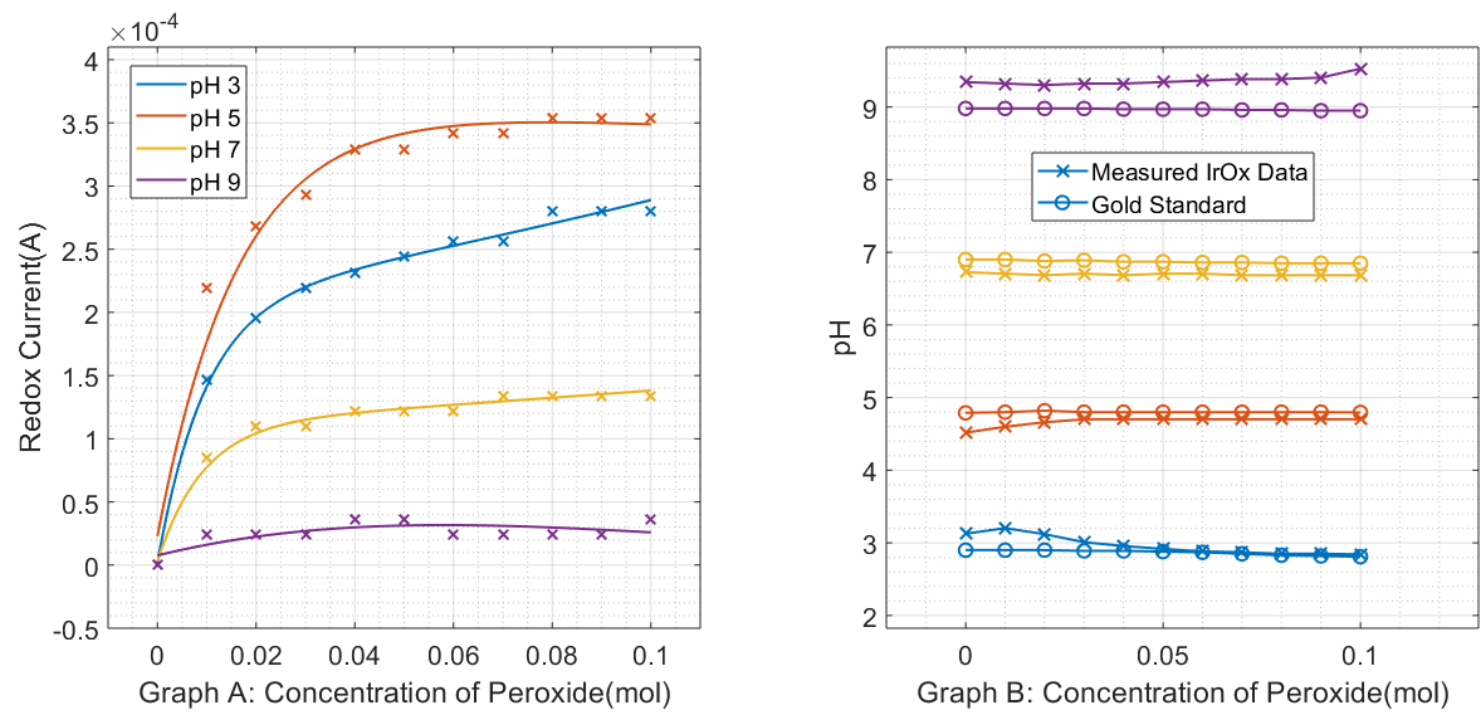

Fig. 7. Graph A: Combined amperometric readings for cell current measurements across a range of concentrations for various $\mathrm{pH}$ buffers with background current removed. Graph B: Combined potentiometric measurements of $\mathrm{pH}$ values of buffers for varying concentration of hydrogen peroxide compared against a measured control.

looking at how much this cell current would cause the actual readings to differentiate or deviate from the gold standard.

Fig. 7A demonstrates that additional $\mathrm{H}_{2} \mathrm{O}_{2}$ concentrations increase the cell current. The large increment in cell current at lower $\mathrm{pH}$ values $(3,5)$ compared to the higher $\mathrm{pH}$ values $(7,9)$ is likely due to all $\mathrm{H}^{+}$ions introduced by the $\mathrm{H}_{2} \mathrm{O}_{2}$ reacting with the $\mathrm{OH}^{-}$ions in the $\mathrm{pH} 9$ solution to form water - which is neutral.

Another important observation that can be made is that the largest variations in cell current occurs for the lower $\mathrm{H}_{2} \mathrm{O}_{2}$ concentrations at $\mathrm{pH} 5$ and 7. This is reflected in Fig. 7B, where the offset for the $\mathrm{pH} 3$ and $\mathrm{pH} 5$ potentiometric readings are largest for the first 2 samples. However, as additional $\mathrm{H}_{2} \mathrm{O}_{2}$ concentrations are added, the combined potentiometric readings follow the gold standard.

Table I shows that the difference in $\mathrm{pH}$ between the control and experiment for $\mathrm{pH} 9$ is the highest, although it remains relatively stable throughout. In comparison, the difference for $\mathrm{pH} 3$ is the lowest, although its variation is the highest among the compared buffers (as seen from its standard deviation of $0.103)$. The variation in this difference could be attributed to the varying cell current, and serves to relate the theory that the cell current would affect potentiometric readings. However, for values of cell current in the magnitude of $10^{-4} \mathrm{~A}$, it is clear from the results that the variation is minuscule. This leads to the conclusion that potentiometric readings are capable of accurately reading values in the presence of cell currents, and is demonstrated for cell currents up to $10^{-4} \mathrm{~A}$.

\section{CONCLUSION}

This work shows that concurrent measurements of potentiometry and amperometry is possible with a shared reference electrode. It demonstrates clear similarities in their trends, for amperometry and in particular for potentiometry even with a cell current applied. The high correlation values, as well as similar sensitivities of the sensors showcase that the combined configuration is equivalent to their conventional counterparts.

The efficacy of concurrent sensing is also showcased through the novel design of a test that varies $\mathrm{pH}$ and $\mathrm{H}_{2} \mathrm{O}_{2}$ concentrations concurrently. The presence of cell current is acknowledged, and shown that at levels such as $10^{-4} \mathrm{~A}$, the potentiometric readings are relatively unaffected.

For future work, a CMOS IC based on the above topology has been designed and will be tested with 4 on-chip electrodes [42]. This IC will be used to further analyse the concepts of concurrent sensing and prove its viability in extremely small packages.

\section{REFERENCES}

[1] A. J. Bandodkar and J. Wang, "Non-invasive wearable electrochemical sensors: a review," Trends in biotechnology, vol. 32, no. 7, pp. 363-371, 2014.

[2] S. Carrara, Bio/CMOS interfaces and co-design. Springer Science \& Business Media, 2012.

[3] P. Yager, G. J. Domingo, and J. Gerdes, "Point-of-care diagnostics for global health," Annual review of biomedical engineering, vol. 10, 2008.

[4] E. Bakker and E. Pretsch, "Trace level potentiometry," Analytical Chemistry, vol. 74, pp. 420A-426A, 2002.

[5] J. Ding and W. Qin, "Recent advances in potentiometric biosensors," TrAC Trends in Analytical Chemistry, p. 115803, 2020.

[6] N. Moser, C. L. Leong, Y. Hu, C. Cicatiello, S. Gowers, M. Boutelle, and P. Georgiou, "Complementary metal-oxide-semiconductor potentiometric field-effect transistor array platform using sensor learning for multi-ion imaging," Analytical chemistry, vol. 92, no. 7, pp. 5276-5285, 2020.

[7] M. Ciobanu, D. E. Taylor, J. P. Wilburn, and D. E. Cliffel, "Glucose and lactate biosensors for scanning electrochemical microscopy imaging of single live cells," Analytical chemistry, vol. 80, no. 8, pp. 2717-2727, 2008.

[8] R. Baron and J. Saffell, "Amperometric gas sensors as a low cost emerging technology platform for air quality monitoring applications: A review," ACS sensors, vol. 2, no. 11, pp. 1553-1566, 2017.

[9] M. Diabetes, "Medtronic enlite glucose sensors," 10 January 2020. [Online]. Available: https://www.medtronic-diabetes.co.uk/continuousglucose-monitoring/enlite-sensor

[10] H. Li, X. Liu, L. Li, X. Mu, R. Genov, and A. J. Mason, "Cmos electrochemical instrumentation for biosensor microsystems: A review," Sensors, vol. 17, no. 1, p. 74, 2017. 
[11] P. Panjan, E. Ohtonen, P. Tervo, V. Virtanen, and A. Sesay, "Shelf life of enzymatic electrochemical sensors," Procedia technology, vol. 27, pp. 306-308, 2017.

[12] S. T. Sanjay, G. Fu, M. Dou, F. Xu, R. Liu, H. Qi, and X. Li, "Biomarker detection for disease diagnosis using cost-effective microfluidic platforms," Analyst, vol. 140, no. 21, pp. 7062-7081, 2015.

[13] S. K. Vashist, "Point-of-care diagnostics: Recent advances and trends," 2017.

[14] S. Baliga, S. Muglikar, R. Kale et al., "Salivary ph: A diagnostic biomarker," Journal of Indian Society of Periodontology, vol. 17, no. 4, p. 461, 2013.

[15] S. Kumar, S. Padmashree, and R. Jayalekshmi, "Correlation of salivary glucose, blood glucose and oral candidal carriage in the saliva of type 2 diabetics: A case-control study," Contemporary clinical dentistry, vol. 5, no. 3, p. 312, 2014.

[16] Y. H. Lee, O. Y. Kweon, H. Kim, J. H. Yoo, S. G. Han, and J. H. Oh, "Recent advances in organic sensors for health self-monitoring systems," Journal of Materials Chemistry C, vol. 6, no. 32, pp. 8569-8612, 2018.

[17] A. J. Bard, L. R. Faulkner, J. Leddy, and C. G. Zoski, Electrochemical methods: fundamentals and applications. wiley New York, 1980, vol. 2.

[18] J. E. Choi, J. N. Waddell, K. Lyons, and J. A. Kieser, "Intraoral ph and temperature during sleep with and without mouth breathing." Journal of oral rehabilitation, vol. 43 5, pp. 356-63, 2016.

[19] M. J. Schöning, R. Krause, K. Block, M. Musahmeh, A. Mulchandani, and J. Wang, "A dual amperometric/potentiometric fia-based biosensor for the distinctive detection of organophosphorus pesticides," Sensors and Actuators B: Chemical, vol. 95, no. 1-3, pp. 291-296, 2003.

[20] K. Brunt, "Comparison between the performance of an electrochemical detector flow cell in a potentiometric and an amperometric measuring system using glucose as a test compound," Analyst, vol. 107, no. 1279, pp. 1261-1271, 1982.

[21] A. Sun, A. Venkatesh, and D. A. Hall, "A multi-technique reconfigurable electrochemical biosensor: Enabling personal health monitoring in mobile devices," IEEE transactions on biomedical circuits and systems, vol. 10, no. 5, pp. 945-954, 2016.

[22] H. Jiang, X. Zhou, S. Kulkarni, M. Uranian, R. Seenivasan, and D. A. Hall, "A sub-1 $\mu$ w multiparameter injectable biomote for continuous alcohol monitoring," in 2018 IEEE Custom Integrated Circuits Conference (CICC). IEEE, 2018, pp. 1-4

[23] A. Cavallini, C. Baj-Rossi, S. Ghoreishizadeh, G. De Micheli, and S. Carrara, "Design, fabrication, and test of a sensor array for perspective biosensing in chronic pathologies," in 2012 IEEE Biomedical Circuits and Systems Conference (BioCAS). IEEE, 2012, pp. 124-127.

[24] Y. Xu, C. Li, W. Mei, M. Guo, and Y. Yang, "Equivalent circuit models for a biomembrane impedance sensor and analysis of electrochemical impedance spectra based on support vector regression," Medical \& biological engineering \& computing, vol. 57, no. 7, pp. 1515-1524, 2019.

[25] M. Sharma, A. Gardner, H. Strathman, D. Warren, J. Silver, and R. Walker, "Acquisition of neural action potentials using rapid multiplexing directly at the electrodes," Micromachines, vol. 9, p. 477, 09 2018.

[26] P. Steegstra and E. Ahlberg, "Influence of oxidation state on the ph dependence of hydrous iridium oxide films," Electrochimica acta, vol. 76, pp. 26-33, 2012.

[27] Libretexts. (2020, Jan) 1.7: Ion Selective Electrode Analysis. [Online]. Available: https://chem.libretexts.org/Bookshelves/AnalyticalChemistry

[28] E. Britannica, "Faraday's law of electrochemistry," 4 April 2020. [Online]. Available: https://www.britannica.com/science/Faradays-lawsof-electrolysis

[29] I. Helm, L. Jalukse, and I. Leito, "Measurement uncertainty estimation in amperometric sensors: a tutorial review," Sensors, vol. 10, no. 5, pp. 4430-4455, 2010.

[30] M. W. Shinwari, D. Zhitomirsky, I. A. Deen, P. R. Selvaganapathy, M. J. Deen, and D. Landheer, "Microfabricated reference electrodes and their biosensing applications," Sensors, vol. 10, no. 3, pp. 1679-1715, 2010.

[31] S. Yang, T. Chen, Y. Wang, Z. Peng, and W. G. Wang, "Electrochemical analysis of an anode-supported sofc," Int. J. Electrochem. Sci, vol. 8 , no. 2, pp. 2330-2344, 2013.

[32] É. Pergel, R. E. Gyurcsányi, K. Tóth, and E. Lindner, "Picomolar detection limits with current-polarized pb2+ ion-selective membranes," Analytical chemistry, vol. 73, no. 17, pp. 4249-4253, 2001.

[33] E. Lindner, R. E. Gyurcsányi, and R. P. Buck, "Tailored transport through ion-selective membranes for improved detection limits and selectivity coefficients," Electroanalysis: An International Journal Devoted to Fundamental and Practical Aspects of Electroanalysis, vol. 11, no. 10-11, pp. 695-702, 1999.
[34] W. E. Morf, M. Badertscher, T. Zwickl, N. F. de Rooij, and E. Pretsch, "Effects of controlled current on the response behavior of polymeric membrane ion-selective electrodes," Journal of Electroanalytical Chemistry, vol. 526, no. 1-2, pp. 19-28, 2002.

[35] C. Instruments. (2020, April) CHI111 Ag/AgCl Reference Electrode w/ porous Teflon Tip. [Online]. Available: https://www.chinstruments.com/accessories.shtml

[36] Dropsens. (2020, April) Dropsens 550 Screen Printed Electrode. [Online]. Available: http://www.dropsens.com

[37] M. Wang, S. Yao, and M. Madou, "A long-term stable iridium oxide ph electrode," Sensors and Actuators B: Chemical, vol. 81, no. 2-3, pp. 313-315, 2002.

[38] Sentron. (2020, April) Sentron Si 400 pH Meter. [Online]. Available: http://www.sentron.nl

[39] C. Instruments. (2020, April) CH Instruments Model 700E Bipotentiostat. [Online]. Available: https://www.chinstruments.com/chi700.shtml

[40] T. Instruments. (2020, April) TI LMC 6042 Potentiostat. [Online]. Available: https://www.ti.com/product/LMC6042

[41] D. Harvey et al., "Analytical chemistry 2.0," 2018.

[42] D. Ma, S. S. Ghoreishizadeh, and P. Georgiou, "Dapper: A low power, dual amperometric and potentiometric single-channel front end," IEEE International Symposium on Circuits and Systems 2020.

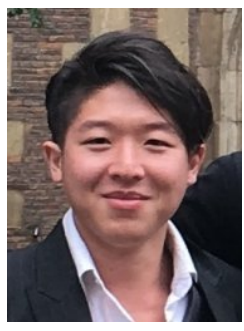

Daryl Ma (Student Member, IEEE) is currently a $\mathrm{PhD}$ candidate in the Centre for Bio-Inspired Technology. He graduated from Imperial College in 2017 with a M.Eng degree in Electrical and Electronic Engineering.

His research interests involves the wireless powering of electrochemical sensors, and spans the fields of inductive coupling and RF systems. This has applications for healthcare wearables, where the aim is to make devices smaller, and also more reliable. He was a recipient of the best student paper award at BioCAS 2017.

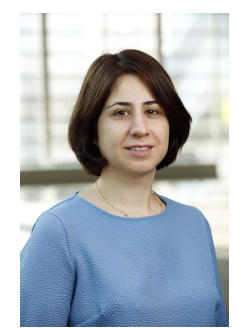

Sara S. Ghoreishizadeh (Member, IEEE) is a lecturer (Assistant Professor) in Wearable Technologies at University College London. She received a PhD from Ecole Polytechnique Federale de Lausanne (EPFL) in 2015 and was with the Dept. of Electrical and Electronic Engineering, Imperial College as a Junior Research Fellow during Apr 2015 to Oct 2018. She received her BSc and MSc degrees (both with distinction) in Electrical Engineering and Microelectronics from Sharif University of Technology in 2007 and

2009, respectively.

She is also a Fellow of the Higher Education Academy (FHEA) and a member of IEEE, and IEEE CAS, SSC and EMB societies. She is also a member of several technical programmes and review committees of IEEE conferences including BioCAS 2017-2019, ICECS 2016-2020 and ISCAS 2018-2020, and a member of the IEEE BioCAS technical committee. She has also been elected to the IEEE CAS Board of Governors (BoG) for the period of 2020-2022. Her current research interests include analogue/mixed-signal IC design and the monolithic integration of biosensors with microelectronics (Lab-on-Chip) 


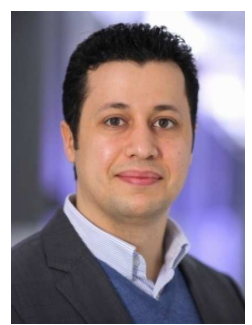

Pantelis Georgiou (Senior Member, IEEE) received the M.Eng. degree in electrical and electronic engineering and the Ph.D. degree from Imperial College London (ICL), London, U.K., in 2004 and 2008, respectively. He is currently a Reader with the Department of Electrical and Electronic Engineering, ICL, where he is also the Head of the Centre for Bio-Inspired Technology, Bio-Inspired Metabolic Technology Laboratory. His research interests include bio-inspired circuits and systems, CMOS-based lab-on-chip technologies, and application of microelectronic technology to create novel medical devices. He has made significant contributions to integrated chemical-sensing systems in CMOS, conducting pioneering work on the development of ISFET sensors, which has enabled applications, such as point-of-care diagnostics and semiconductor genetic sequencing and has also developed the first bio-inspired artificial pancreas for treatment of Type I diabetes using the silicon-beta cell. He is a member of the IET and serves on the BioCAS and Sensory Systems technical committees of the IEEE CAS Society. He received the IET Mike Sergeant Medal of Outstanding Contribution to Engineering in 2013. In 2017, he also received the IEEE Sensors Council Technical Achievement Award. $\mathrm{He}$ is also the CAS representative on the IEEE Sensors council and the IEEE Distinguished Lecturer in Circuits and Systems. 